\title{
Morphological study of the anthropoid thoracic cage: scaling of thoracic width and an analysis of rib curvature
}

\author{
Miyuki Kagaya $\cdot$ Naomichi Ogihara
}

Masato Nakatsukasa

Published online: 16 January 2008

(C) Japan Monkey Centre and Springer 2008

\section{Erratum to: Primates}

DOI 10.1007/s10329-007-0064-z

We regret that the original version of this article contained an error in Fig. 5 regarding the style of the lines of the third rib. The corrected figure is shown here with the scale.

Fig. 5 Average shapes of ribs on the transverse plane normalized by rib length in five groups of primates
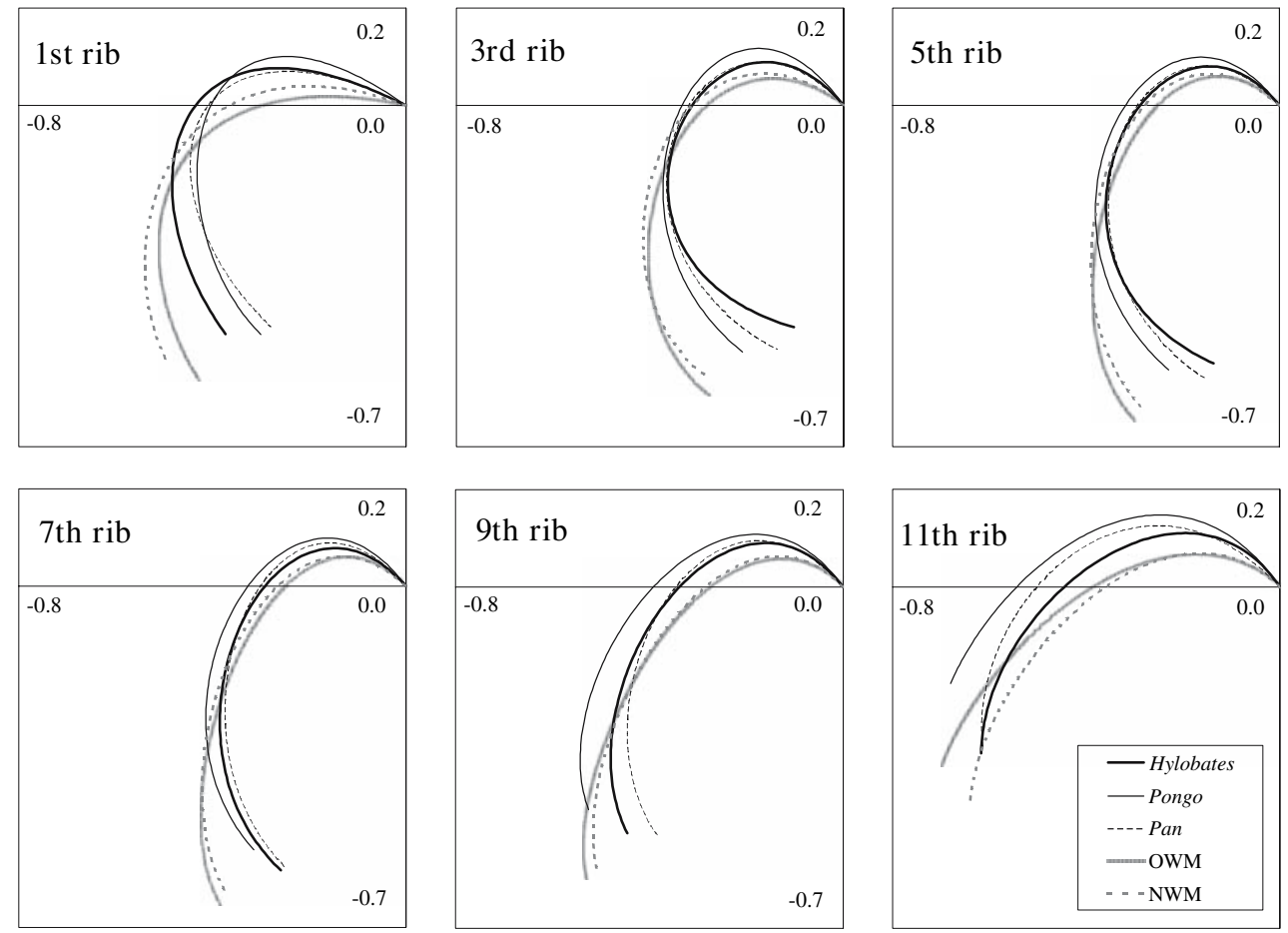

The online version of the original article can be found under doi:10.1007/s10329-007-0064-z.

M. Kagaya $(\bowtie) \cdot$ N. Ogihara $\cdot$ M. Nakatsukasa

Laboratory of Physical Anthropology, Department of Zoology,

Graduate School of Science, Kyoto University, Sakyo,

Kyoto 606-8502, Japan

e-mail: kagaya@anthro.zool.kyoto-u.ac.jp 